\title{
Thyrotoxicosis Factitia: A Rare Cause of Junctional Rhythm and Cardiac Arrest
}

Iqra Iqbal $^{1}$, Muhammad AtiqueAlam Khan ${ }^{1}$, Waqas Ullah ${ }^{1}$, Dina Nabwani ${ }^{2}$

${ }^{1}$ Abington Hospital - Jefferson Health, Abington, PA, USA; Bayshore Medical Center, Holmdel, NJ, USA.

\section{INTRODUCTION}

Little is known about the types of arrhythmias due to exogenous thyroxine. We present an unusual set of cardiac arrhythmias in a fairly healthy athlete who succumbed to the severe complications of exogenous thyroxine.

\section{CASE DESCRIPTION}

A 50-year-old female athlete was brought into the emergency department (ED) after a witnessed car accident. She was pulseless, so she was intubated and resuscitated in the field.

In the ER, she was found to be in a junctional rhythm with diffuse ST depressions. Initial blood workup was unremarkable. Drug screen was negative. Arterial blood gas (ABG) showed respiratory acidosis.

The only medication the patient was taking was thyroxine for her hypothyroidism.
She was known by family to be abusing it for weight loss purposes. Her serum thyroxine (T4) levels turned out to be elevated (365ng) and TSH was less than 0.02 .

\section{DESCISION-MAKING}

The patient went into cardiac arrest again and was persistently in the junctional rhythm.(Figure 1) This eventually led her into severe bradycardia and cardiogenic shock. She was later resuscitated twice again. However, by that point, the patient had pinpoint pupils and brain imaging was consistent with anoxic brain injury

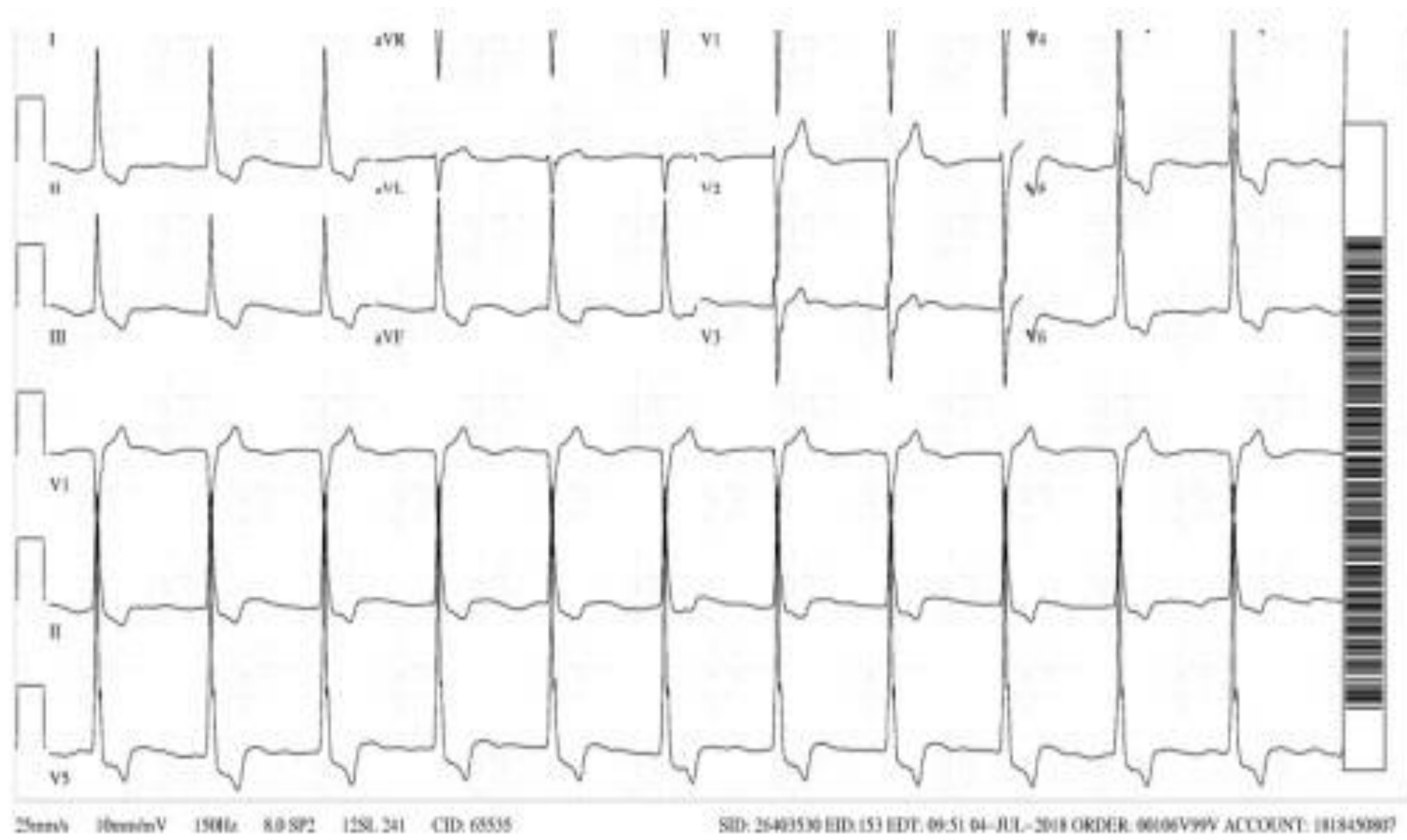

Figure 1: ECG showing the junctional rhythm with some evidence of left ventricular hypertrophy.

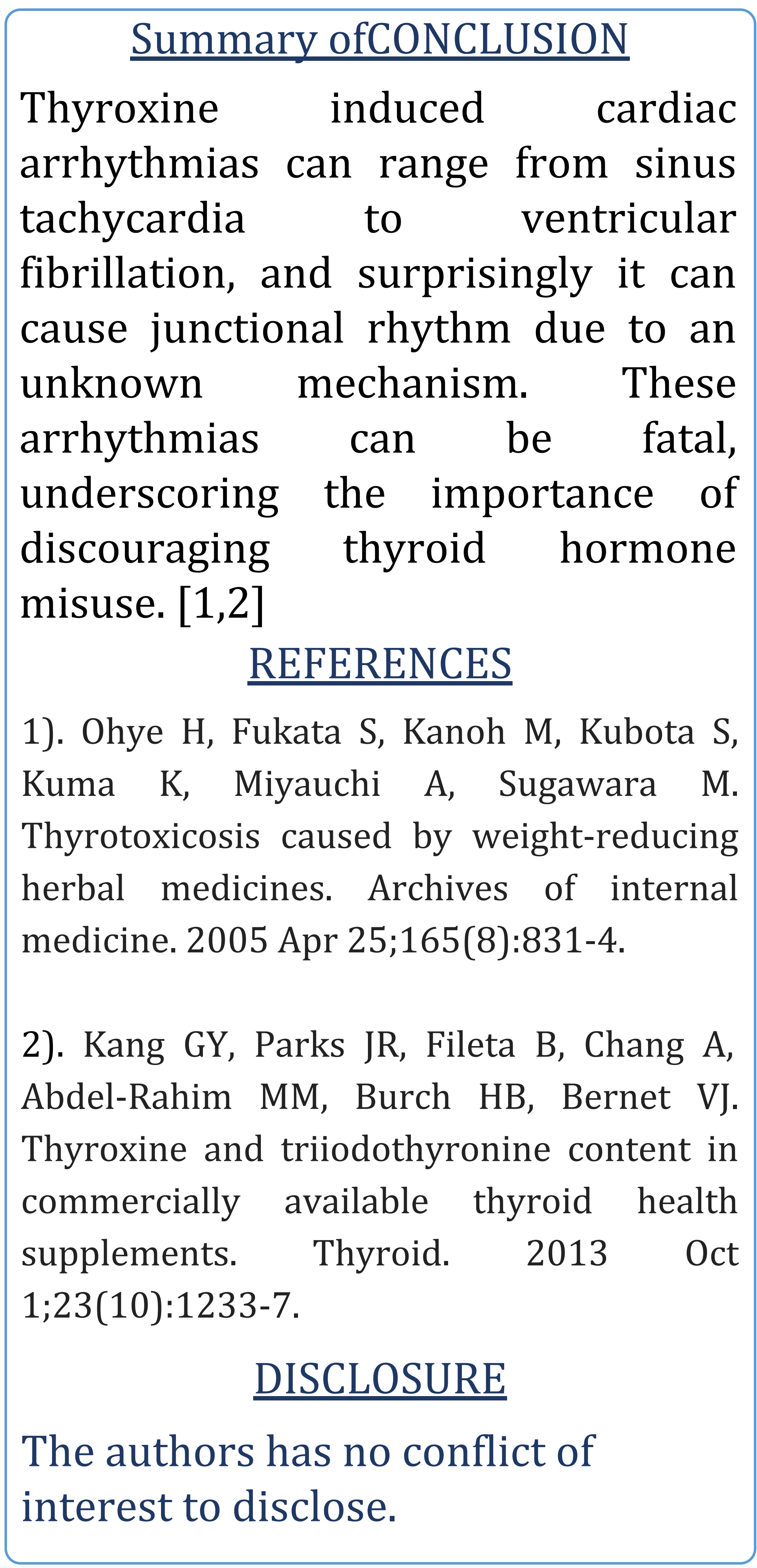

